\title{
Asymptomatic malaria and hepatitis B do not influence cytokine responses of persons involved in chronic sedentary activities
}

Nsoh Godwin Anabire ${ }^{1,2}$, Paul Armah Aryee ${ }^{3}$, Zulka Ziblim², Jonathan Suurbaar ${ }^{1}$, Felix Ansah ${ }^{1}$ and Gideon Kofi Helegbe ${ }^{1,2^{*}}$ (i)

\begin{abstract}
Background: Chronic Sedentary lifestyles have been linked to increased odds of stress, elevated anxiety and diminished wellbeing, inducing cytokine production and predispose to hypertension and other cardiovascular diseases. In endemic areas, Plasmodium falciparum and hepatitis B virus (HBV) infections can trigger pro-inflammatory cytokine responses. However, the impact of these infections on cytokine response profiles in individuals engaged in chronic sedentary activities is unknown. This study was aimed at addressing these concerns using a predominantly sedentary population of traders in the Tamale metropolis of Ghana.

Method: Four hundred respondents were categorized, based on their number of working years ( $<$ or $\geq 5$ years) and number of working hours per day $(<$ or $\geq 10 \mathrm{~h})$, into sedentary $(\geq 5$ years $+\geq 10 \mathrm{~h})$ and non-sedentary $(\geq$ 5 years $+<10 \mathrm{~h},<5$ years $+\geq 10 \mathrm{~h}$ and $<5$ years $+<10 \mathrm{~h}$ ) groups. The participants were tested for $P$. falciparum and HBV infections using polymerase chain reaction. Blood pressure and cytokines responses were measured. Associations and comparison analysis between variables were determined, and test statistics with $p<0.05$ were considered statistically significant.

(Continued on next page)
\end{abstract}

\footnotetext{
* Correspondence: ghelegbe@uds.edu.gh

${ }^{1}$ West African Centre for Cell Biology of Infectious Pathogens (WACCBIP),

University of Ghana, P. O. Box LG 54, Legon, Accra, Ghana

2Department of Biochemistry \& Molecular Medicine, School of Medicine,

University for Development Studies, P. O. Box TL, 1883 Tamale, Ghana

Full list of author information is available at the end of the article
}

(c) The Author(s). 2020 Open Access This article is licensed under a Creative Commons Attribution 4.0 International License, which permits use, sharing, adaptation, distribution and reproduction in any medium or format, as long as you give appropriate credit to the original author(s) and the source, provide a link to the Creative Commons licence, and indicate if changes were made. The images or other third party material in this article are included in the article's Creative Commons licence, unless indicated otherwise in a credit line to the material. If material is not included in the article's Creative Commons licence and your intended use is not permitted by statutory regulation or exceeds the permitted use, you will need to obtain permission directly from the copyright holder. To view a copy of this licence, visit http://creativecommons.org/licenses/by/4.0/ The Creative Commons Public Domain Dedication waiver (http://creativecommons.org/publicdomain/zero/1.0/) applies to the data made available in this article, unless otherwise stated in a credit line to the data. 


\begin{abstract}
(Continued from previous page)
Results: Infection status included: un-infected (93.5\%), P. falciparum mono-infected (1.0\%), HBV mono-infected (3.0\%) or P. falciparum /HBV co-infected (2.5\%). Majority of the participants, $57.0 \%(n=228)$ were involved in chronic sedentary life style. That notwithstanding, sedentary lifestyle was independent of the infection groups $\left(X^{2}=7.08, p=0.629\right)$. Hypertension was diagnosed in $53.8 \%$ of respondents and was independent of infection status $\left(X^{2}=6.33, p=0.097\right)$. Pro-inflammatory (TNF- $a, I L-1 \beta, I L-6, I L-8$ and IL-12) and anti-inflammatory (IL-10, IL7 and IL-13) cytokine responses were similar among individuals with different sedentary working time and between hypertensive and non-hypertensive individuals ( $p>0.05$ for all comparisons). Among individuals with different infection status, pro-inflammatory (TNF- $\alpha ; p=0.290, I L-1 \beta ; p=0.442, I L-6 ; p=0.686$, IFN- $\gamma ; p=0.801$, IL-8; $p=0.546, I L-12 ; p=0.154)$ and anti-inflammatory $(I L-10 ; p=0.201, I L-7 ; p=0.190, I L-13 ; p=0.763)$ cytokine responses were similar.

Conclusion: Our data suggest that asymptomatic infections of P. falciparum and HBV together with a high prevalence of hypertension did not have any significant impact on cytokine response profiles among predominantly sedentary traders in the Tamale metropolis of Ghana.
\end{abstract}

Keywords: Sedentary lifestyle, Hypertension, Cytokines, Malaria, Hepatitis B

\section{Background}

Behavioural or lifestyle activities such as unhealthy diet, physical inactivity, smoking and harmful use of alcohol are recognized as important risk factors of cardiovascular diseases (CVDs) [1, 2]. The effects of these behavioural risk factors may show up in individuals as elevated blood pressure or blood glucose or blood lipids, as well as overweight and obesity $[1,2]$. Notwithstanding, the involvement of sedentary lifestyle as a risk factor for hypertension and CVDs cannot be overruled [3, 4]. In particular, prolonged sedentary time can impact upon inflammation [57], and is associated with elevated pro-inflammatory and reduced anti-inflammatory cytokine responses [5, 8-10]. Cytokine imbalances, specifically a shift towards proinflammatory responses, plays a key role in the pathogenesis of hypertension and CVDs [11].

Sub-Saharan African countries are faced with a huge burden of infectious diseases such as malaria and hepatitis B. Particularly, in malaria endemic settings, it is common to find adults with asymptomatic Plasmodium falciparum infections who have developed semi-immunity due to exposure to the parasite [12]. Also, it is common to find adults with chronic hepatitis B virus (HBV) infection who experience no disease symptoms [13]. Though current evidence shows no links between P. falciparum or HBV and CVDs $[14,15]$, both pathogenic diseases majorly trigger inflammatory immune responses [16], and could exacerbate inflammatory responses in adults involved in chronic sedentary lifestyle, which may predispose them to hypertension and CVD.

In many endemic countries including Ghana, traders are observed to be engaged in stress related sedentary activities as they sit for long hours as part of their work [17]. This current study evaluates the impact of carriage of HBV and/or P. falciparum on cytokine profiles of this group of people and their risk to developing hypertension. The data generated would be useful in informing the implicit link of these infections to the risk of hypertension and CVDs.

\section{Methods}

\section{Study design, study site and population}

A cross-sectional study was conducted from July to September 2017, on traders in the Tamale metropolis, which is the Northern Regional capital of Ghana. Adult traders (18 years and above) who worked predominantly seated were recruited into the study. The Participants were recruited conveniently after close of work, and the numbers obtained within the sampling period was taken as the sample size.

\section{Data collection and processing}

Socio-demographic data (including; age, sex, marital status, educational status, occupational information) and life style behaviours (intake of alcohol, smoking and selfmedication) of each study participant was collected after consent was obtained, using a standardized questionnaire (Supplementary file). Qualified enumerators were given a one-day training, and questionnaire was pretested among a set of traders who did not participate in the study. The participants were categorized into young adults ( $18-35$ years), middle aged adults ( $36-55$ years) and the aged (above 55 years) [18].

Blood pressure (BP) of each participant was measured using a semi-automated (digital) sphygmomanometer, MaRS ${ }^{\bullet}$ model MS-702 (Mars Medical Products Co., Ltd., Taiwan). The average of two BP readings, first reading taken at the close of work and second reading taken the next morning prior to start of work, was used in the analysis. BP was categorized as normal $(<120 /<80$ $\mathrm{mmHg}$ ), elevated $(120-129 /<80 \mathrm{mmHg})$ or hypertension stage $1(130-139 / 80-89 \mathrm{mmHg})$ and hypertension stage 
2 ( $\geq 140 / \geq 90 \mathrm{mmHg})$ [19]. The proxy for assessing chronic sedentary time among the respondents was based on mainly sitting/seated for long hours (beyond the normal working hours, $\geq 10 \mathrm{~h}$ per day), and being involved in the work for a longer period ( $\geq 5$ years).

\section{Laboratory investigations}

Venous blood sample $(5 \mathrm{~mL})$ was collected from each participant by a qualified phlebotomist into an anticoagulant (EDTA) tube, and used for laboratory investigations.

Hemoglobin $(\mathrm{Hb})$ levels of participants were measured using Mission ${ }^{\circ}$ Plus $\mathrm{Hb}$ meter (ACON Laboratories, Inc., San Diego, USA). Anemia was diagnosed by $\mathrm{Hb}<12 \mathrm{~g} / \mathrm{dl}$ for non-pregnant females and $\mathrm{Hb}<13 \mathrm{~g} / \mathrm{dl}$ for males [20].

Participants were screened for malaria and hepatitis B using CareStart ${ }^{\text {Tw }}$ HRP-2 rapid diagnostic test kit (Access Bio Inc., New Jersey, USA) and hepatitis B surface antigen (HbsAg) test strips (Intec Products Inc., Xiamen, China) respectively. The presence or absence of plasmodium infection in each participant was confirmed using a nested polymerase chain reaction (PCR) assay [21, 22]. The presence or absence of HBV infection in each participant was also confirmed by a previously described PCR assay [23]. The PCR reagents, reaction volumes and cycling conditions were the same as we have previously reported [24].

\section{Cytokine analysis}

HSTCMAG28SPMX13 Milliplex MAP kit (Merck, Darmstadt, Germany) was used to assay serum levels of nine cytokines; tumor necrosis factor alpha (TNF- $\alpha$ ), interleukin (IL)-1 $\beta$, IL-6, interferon gamma (IFN- $\gamma$ ), IL-12, IL-8, IL10, IL-7, and IL-13, following the manufacturer's protocol. The quantification was done on the xMAP Technology platform (Luminex Corporation, Austin, USA). The values of the quality controls and standards were within the ranges provided by the kit. Samples were assayed in duplicates and those with percentage coefficient of variation < $15 \%$ were included in the data analysis.

\section{Statistical analysis}

Microsoft Excel 2016 (Microsoft Corporation, Redmond, USA) was used for data entry and proofreading. SPSS Version 20 (IBM Corporation, Chicago, USA) and GraphPad Prism 6 (GraphPad Software Inc.) were used to analyze the data. Median and interquartile range (IQR) was used to present continuous variables and comparisons were done by Krulskal-Wallis or MannWhitney test. On the other hand, counts and percentages were used to describe categorical variables, and comparison analysis were done using Fisher's exact test or Pearson's chi-square test. $P$-value $<0.05$ was considered as statistically significant for all test statistics.

\section{Results}

\section{Questionnaire response rate and socio-demographic} information

The questionnaire was administered to a total of 400 traders. All the participants responded to the questionnaire $(100 \%$ response rate). Majority $(46.0 \%, n=224)$ of the respondents were young adults $(18-35$ years, Additional file 1). Also, $78.5 \%(n=314)$ of them were married, and majority $(64.5 \%, n=258)$ had attained some level of formal education (Additional file 1).

\section{Behavioral/life style determinants among study participants}

Majority $(82.5 \% n=330)$ of respondents were involved in trading for $\geq 5$ years (Table 2). Many $(69.8 \%, n=279)$ worked, predominantly sitting/seated beyond the normal work hours per day ( $\geq 10 \mathrm{~h}$, Table 2$)$. A smaller proportion of the participants responded to intake of alcohol $(3.0 \%$. $n=12)$ and smoking (2.5\%. $n=10)$ (Additional file 2). Though all the participants responded to not taking any medications or drugs in the last month prior to the study, more than half $(50.5 \%, n=202)$ were in the habit of selfmedication whenever they were sick such as having chest and bodily pains (Additional file 2). The self-medicated drugs were mainly antimalarials and pain killers. Close to a half $(44.5 \%, n=178)$ of the respondents were diagnosed with anemia (Supplementary Table 2). Also, more than half were diagnosed with hypertension (type 1 and 2) (53.8\%, $n=215$, Additional file 2).

\section{Sedentary and infection groups}

The participants were categorized into 4 groups based on their number of working years $(<$ or $\geq 5$ years $)$ and number of working hours per day $(<$ or $\geq 10 \mathrm{~h})$. The predominantly sedentary participants included those in $\geq 5$ years $+\geq 10 \mathrm{~h}$ group $(57.0 \%, n=228)$ and the nonsedentary group included those in $\geq 5$ years $+<10 \mathrm{~h}$ group (25.5\%, $n=102),<5$ years $+\geq 10 \mathrm{~h}$ group $(12.8 \%$, $n=51)$, and $<5$ years $+<10 h$ group $(4.8 \%, n=19)$. Based on the PCRs, the infection status of the participants included un-infected $(93.5 \%, n=374)$, P. falciparum mono-infected $(1.0 \%, n=4)$, HBV mono-infected $(3.0 \%, n=12)$ or P. falciparum/HBV co-infected $(2.5 \%$, $\mathrm{n}=10)$. In the sedentary group, $212(53.0 \%)$ were uninfected, $2(0.5 \%)$ had $P$. falciparum mono-infection, 7 (1.8\%) had HBV mono-infection while $7(1.8 \%)$ had $P$. falciparum/HBV co-infection (Table 1). Sedentary lifestyle was independent of the infection groups $\left(\chi^{2}=7.08\right.$, $p=0.629$, Table 1).

\section{Socio-demographic information and determinants of stress among infection groups}

The proportions of young adults, middle age adults and the aged were similar among the different infection 
Table 1 Association between Sedentary life style and Infection status

\begin{tabular}{|c|c|c|c|c|c|}
\hline \multirow[t]{2}{*}{ Infection status } & \multicolumn{4}{|c|}{ Sedentary and non-sedentary groups } & \multirow[t]{2}{*}{$\left(X^{2}\right),{ }^{*} p$} \\
\hline & $\begin{array}{l}>=5 \text { years }+>=10 \mathrm{~h} \mathrm{n} \\
(\%)\end{array}$ & $\begin{array}{l}>=5 \text { years }+<10 \mathrm{~h} \mathrm{n} \\
(\%)\end{array}$ & $\begin{array}{l}<5 \text { years }+>=10 \mathrm{~h} \mathrm{n} \\
(\%)\end{array}$ & $\begin{array}{l}<5 \text { years }+<10 \mathrm{~h} \mathrm{n} \\
(\%)\end{array}$ & \\
\hline Un-infected & $212(53.0)$ & $96(24.0)$ & $50(12.5)$ & $16(4.0)$ & \multirow{4}{*}{$\begin{array}{l}7.08 \\
{ }^{*} 0.629\end{array}$} \\
\hline $\begin{array}{l}\text { P. falciparum mono- } \\
\text { infection }\end{array}$ & $2(0.5)$ & $1(0.3)$ & $0(0.0)$ & $1(0.3)$ & \\
\hline HBV mono-infection & $7(1.8)$ & $3(0.8)$ & $1(0.3)$ & $1(0.3)$ & \\
\hline Co-infected & $7(1.8)$ & $2(0.5)$ & $0(0.0)$ & $1(0.3)$ & \\
\hline
\end{tabular}

* analyzed using Pearson's chi-square test or Fisher's exact test

$x^{2}=$ Chi-square value

$p$ significant at $<0.05$ (2-tailed)

$\mathrm{n}(\%)$ : numbers and proportions

statuses $\left(\chi^{2}=12.17, p=0.058\right.$, Table 2$)$. Similarly, no differences in sex $\left(\chi^{2}=4.89, p=0.108\right)$, marital status $\left(\chi^{2}=\right.$ 6.98, $p=0.073)$, and educational status $\left(\chi^{2}=3.51, p=\right.$ 0.320 ) were observed among the different infection statuses (Table 2). The proportions of participants with life style factors such as intake of alcohol $\left(\chi^{2}=0.86, p=\right.$
$0.835)$, smoking $\left(\chi^{2}=3.49, p=0.321\right)$ and resort to selfmedications $\left(\chi^{2}=1.70, p=0.636\right)$ were also similar among individuals with different infection status (Table $2)$. Anemia $\left(\chi^{2}=4.03, p=0.258\right)$ and hypertension $\left(\chi^{2}=\right.$ 6.33, $p=0.097$ ) were also independent of infection status (Table 2).

Table 2 Differences in socio-demographic variables and stress determinants among infection groups

\begin{tabular}{|c|c|c|c|c|c|c|}
\hline \multirow[t]{2}{*}{ Category } & \multirow{2}{*}{$\begin{array}{l}\text { Sub- } \\
\text { category }\end{array}$} & \multicolumn{4}{|l|}{ Infection status } & \multirow[t]{2}{*}{$\left(X^{2}\right), * p$} \\
\hline & & $\begin{array}{l}\text { Un-infected n } \\
(\%)\end{array}$ & $\begin{array}{l}\text { P. falciparum mono-infection } \mathrm{n} \\
\text { (\%) }\end{array}$ & $\begin{array}{l}\text { HBV mono-infection } \mathrm{n} \\
\text { (\%) }\end{array}$ & $\begin{array}{l}\text { Co-infected } \mathrm{n} \\
\text { (\%) }\end{array}$ & \\
\hline \multicolumn{7}{|c|}{ Socio-economic variables } \\
\hline \multirow[t]{3}{*}{ Age } & $18-35$ & $202(54.0)$ & $4(100.0)$ & $8(66.7)$ & $10(100.0)$ & \multirow{3}{*}{$\begin{array}{l}12.17 \\
* 0.058\end{array}$} \\
\hline & $36-55$ & $124(33.2)$ & $0(0.0)$ & $3(25.0)$ & $0(0.0)$ & \\
\hline & $56^{+}$ & $48(12.8)$ & $0(0.0)$ & $1(8.3)$ & $0(0.0)$ & \\
\hline \multirow[t]{2}{*}{ Sex } & Male & $255(68.2)$ & $3(75.0)$ & $9(75.0)$ & $10(100.0)$ & \multirow[t]{2}{*}{$4.89,{ }^{*} 0.180$} \\
\hline & Female & $119(31.8)$ & $1(25.0)$ & $3(25.0)$ & $0(0.0)$ & \\
\hline \multirow[t]{2}{*}{ Marital Status } & Yes & $296(79.1)$ & $1(25.0)$ & $9(75.0)$ & $8(80.0)$ & \multirow[t]{2}{*}{$6.98, * 0.073$} \\
\hline & No & $78(20.9)$ & $3(75.0)$ & $3(25.0)$ & $2(20.0)$ & \\
\hline \multirow{2}{*}{$\begin{array}{l}\text { Formal } \\
\text { education }\end{array}$} & Yes & $239(63.9)$ & $4(100.0)$ & $7(58.3)$ & $8(80.0)$ & \multirow[t]{2}{*}{$3.51,{ }^{*} 0.320$} \\
\hline & No & $135(36.1)$ & $0(0.0)$ & $5(41.7)$ & $2(20.0)$ & \\
\hline \multicolumn{7}{|c|}{ Life style determinants } \\
\hline \multirow[t]{2}{*}{ Alcohol intake } & Yes & $12(3.0)$ & $0(0.0)$ & $0(0.0)$ & $0(0.0)$ & \multirow[t]{2}{*}{$0.86, * 0.835$} \\
\hline & No & $388(97.0)$ & $4(100.0)$ & $12(100.0)$ & $10(100.0)$ & \\
\hline \multirow[t]{2}{*}{ Smoking } & Yes & $21(5.6)$ & $1(25.0)$ & $1(8.3)$ & $0(0.0)$ & \multirow[t]{2}{*}{$3.51,{ }^{*} 0.320$} \\
\hline & No & $353(94.4)$ & $3(75.0)$ & $11(91.7)$ & $10(100.0)$ & \\
\hline \multirow[t]{2}{*}{ Self-medication } & Yes & $186(49.7)$ & $2(50.0)$ & $8(66.7)$ & $6(60.0)$ & \multirow[t]{2}{*}{$1.70,{ }^{*} 0.636$} \\
\hline & No & $188(50.3)$ & $2(50.0)$ & $4(33.3)$ & $4(40.0)$ & \\
\hline \multicolumn{7}{|c|}{ Diagnosed anemia and hypertension } \\
\hline \multirow[t]{2}{*}{ Anemia } & Yes & $170(45.5)$ & $0(0.0)$ & $4(33.3)$ & $4(40.0)$ & \multirow[t]{2}{*}{$4.03,{ }^{*} 0.258$} \\
\hline & No & $204(54.5)$ & $4(100.0)$ & $8(66.7)$ & $6(60.0)$ & \\
\hline \multirow[t]{2}{*}{ Hypertensive } & Yes & $254(67.9)$ & $4(100.0)$ & $5(41.7)$ & $8(80.0)$ & \multirow[t]{2}{*}{$6.33,{ }^{*} 0.097$} \\
\hline & No & $120(32.1)$ & $0(0.0)$ & $7(58.3)$ & $2(20.0)$ & \\
\hline
\end{tabular}

*analyzed using Pearson's chi-square test or Fisher's exact test

$\mathrm{X}^{2}=$ Chi-square value

$p$ significant at $<0.05$ (2-tailed)

$\mathrm{n}(\%)$ : numbers and proportions 
Cytokine responses between sedentary and nonsedentary groups

To evaluate the impact of chronic sedentary lifestyle on serum cytokine response levels, pro- and antiinflammatory cytokine responses were compared between the sedentary group; $\geq 5$ years $+\geq 10 \mathrm{~h}$ group $(n=24)$ and the non-sedentary groups; $\geq 5$ years $+<10 \mathrm{~h}$ group $(n=$ $6),<5$ years $+\geq 10$ h group $(n=4)$, and $<5$ years $+<10$ $\mathrm{h}$ group $(n=4)$. The comparison analysis showed similar levels of pro-inflammatory (TNF- $\alpha ; p=0.405$, IL-1 $\beta ; p=$ 0.759 , IL-6; $p=0.608$, IFN- $\gamma ; p=0.890$, IL-8; $p=0.773$, IL-12; $p=0.667$; Table 2) and anti-inflammatory (IL-10; $p=0.342$, IL-7; $p=0.222$, IL-13; $p=0.927$; Table 3) cytokine responses across the four groups.

\section{Cytokine profiles among participants diagnosed with hypertension or anemia}

The influence of hypertension or anemia on serum cytokine profiles among the participants was evaluated. Comparison analysis showed similar levels of proinflammatory (TNF- $\alpha$, IL- $1 \beta$, IL-6, IFN- $\gamma$, IL- 8 and IL12) and anti-inflammatory (IL-10, IL-7 and IL-13) cytokine responses between hypertensive and nonhypertensive participants and between anemic and nonanemic participants $(p>0.05$ for all comparisons, Table 4).

\section{Cytokine profiles among infection groups}

To gain insight into the possible influence of asymptomatic carriage of $P$. falciparum and/or HBV on cytokine responses among the participants, the cytokine responses were compared across the infection statuses. Serum levels of pro-inflammatory cytokines were similar among the participants with different infection status
(TNF- $\alpha ; p=0.290, I L-1 \beta ; p=0.442, I L-6 ; p=0.686, I F N-$ $\gamma ; p=0.801, I L-8 ; p=0.546, I L-12 ; p=0.154$; Table 4). Similarly, no differences in anti-inflammatory cytokine responses were observed across the infection statuses $(I L-10 ; \quad p=0.201, \quad I L-7 ; \quad p=0.190, \quad I L-13 ; \quad p=0.763$; Table 5). Next, the participants stratified into four groups as: those involved in sedentary lifestyle and infected with $P$. falciparum and/or HBV infections, those involved in sedentary life style and Un-infected, those involved in non-sedentary lifestyle and infected with $P$. falciparum and/or HBV infections, and those involved in non-sedentary life style and Un-infected. Comparison analysis showed that levels of the pro- and antiinflammatory cytokine levels were similar across the groups ( $p>0.05$ for all comparisons, Table 6 ).

\section{Discussion}

This current study evaluates the influence of carriage of HBV and P. falciparum infections on cytokine profiles of predominantly sedentary traders in the Tamale metropolis of Ghana. This is from a viewpoint that sedentary activities of traders can predispose them to CVDs $[3,4]$. Amidst various reasons, predisposition to hypertension and CVDs could be ascribed to induction of inflammatory mediators, and strong evidence suggests that prolonged sedentary time impacts upon inflammation [57]. Since HBV and $P$. falciparum infections majorly trigger pro-inflammatory cytokine responses, they could be viewed to increase the risk of hypertension in persons involved in chronic sedentary behaviour.

The study established chronic sedentary behaviour as common among the traders, nevertheless, the behaviour had no significant impact on the cytokine profiles of the traders. In particular, prolonged sedentary time is

Table 3 Comparison of cytokine responses across different lifestyle groups

\begin{tabular}{|c|c|c|c|c|c|}
\hline \multirow{2}{*}{$\begin{array}{l}\text { Parameters } \\
(\mathrm{pg} / \mathrm{mL})\end{array}$} & \multicolumn{4}{|c|}{ Groups of traders based on number of working years and working hours per day } & \multirow[t]{2}{*}{${ }^{*} p$} \\
\hline & $\geq 5$ years $+\geq 10 \mathrm{~h}$ group & $\geq 5$ years $+<10 \mathrm{~h}$ group & $<5$ years $+\geq 10 \mathrm{~h}$ group & $<5$ years $+<10 \mathrm{~h}$ group & \\
\hline \multicolumn{3}{|c|}{ Pro-inflammatory cytokines } & \multicolumn{2}{|l|}{ Median (IQR), n } & \\
\hline TNF-a & $12.12(9.54-15.38) 20$ & $11.35(9.65-11.92) 5$ & $12.69(6.91-14.51) 4$ & $7.13(4.50-12.99) 4$ & 0.405 \\
\hline $\mathrm{IL}-1 \beta$ & $3.47(2.82-4.82) 15$ & $3.52(2.59-5.503) 4$ & $4.56(3.30-5.73) 3$ & $3.37(1.97-4.56) 3$ & 0.759 \\
\hline IL-6 & $34.88(25.83-47.90) 20$ & $28.41(23.06-36.30) 5$ & $39.55(21.62-52.94) 4$ & $25.32(21.62-131.3) 4$ & 0.608 \\
\hline IFN- $\gamma$ & 6.355 (3.94-9.83) 18 & $5.39(3.73-8.44) 5$ & 7.34 (3.69-10.77) 3 & $7.95(3.01-14.20) 4$ & 0.890 \\
\hline IL-8 & $14.21(10.32-36.89) 13$ & $12.16(4.09-20.22) 2$ & $35.94(11.06-404.4) 4$ & $15.93(3.01-24.02) 3$ & 0.773 \\
\hline $\mathrm{IL}-12$ & $4.328(3.07-5.73) 18$ & $4.27(3.45-6.87) 5$ & $2.161(1.79-5.86) 3$ & $3.872(1.82-5.96) 3$ & 0.667 \\
\hline \multicolumn{3}{|c|}{ Anti-inflammatory cytokines } & \multicolumn{2}{|l|}{ Median (IQR), n } & \\
\hline IL-10 & $28.3(14.17-52.01) 18$ & $16.65(14.91-50.76) 4$ & $13.41(5.69-29.60) 4$ & $17.6(15.06-27.41) 3$ & 0.342 \\
\hline IL-7 & $17.72(14.41-23.66) 21$ & $11.83(10.38-24.46) 5$ & $17.98(11.91-35.01) 4$ & $10.93(6.76-17.98) 4$ & 0.222 \\
\hline IL-13 & $27.17(19.65-35.52) 15$ & $27.47(15.37-42.98) 5$ & $23.26(13.22-34.90) 4$ & $26.27(15.59-39.87) 4$ & 0.927 \\
\hline
\end{tabular}

*; analyzed by Krulskal-Wallis test

$p$ significant at $<0.05$ (2-tailed)

$I Q R$ interquartile range

$n$ number of samples 
Table 4 Comparison of cytokine responses among participants with hypertension or anemia

\begin{tabular}{|c|c|c|c|c|c|c|c|}
\hline \multirow{2}{*}{$\begin{array}{l}\text { Cytokines } \\
(\mathrm{pg} / \mathrm{mL})\end{array}$} & \multirow[t]{2}{*}{ Category } & \multicolumn{3}{|c|}{ Hypertension } & \multicolumn{3}{|c|}{ Anemia } \\
\hline & & $\bar{n}$ & Median (IQR) & ${ }^{\# p} p$ & $\mathrm{~N}$ & Median (IQR) & ${ }^{\# p} p$ \\
\hline \multirow[t]{2}{*}{ TNF-a } & Yes & 22 & $11.48(9.75-14.91)$ & 0.281 & 13 & $11.44(10.28-14.77)$ & 0.573 \\
\hline & No & 11 & $9.91(6.74-12.95)$ & & 20 & $11.62(6.82-13.69)$ & \\
\hline \multirow[t]{2}{*}{ IL-1 $\beta$} & Yes & 19 & $3.55(2.82-5.28)$ & 0.563 & 11 & 3.91 (3.30-5.28) & 0.123 \\
\hline & No & 6 & $3.39(2.66-4.57)$ & & 14 & $3.03(2.377-4.57)$ & \\
\hline \multirow[t]{2}{*}{ IL-6 } & Yes & 21 & $40.64(27.78-47.54)$ & 0.113 & 13 & 37.09 (27.78-43.53) & 0.957 \\
\hline & No & 12 & 27.26 (21.49-37.50 & & 20 & $30.43(23.26-52.17)$ & \\
\hline \multirow[t]{2}{*}{$\mathrm{IFN}-\gamma$} & Yes & 21 & $6.62(3.96-10.12)$ & 0.378 & 12 & 7.12 (5.03-9.38) & 0.409 \\
\hline & No & 9 & $6.89(3.59-7.41)$ & & 18 & $6.26(3.60-8.96)$ & \\
\hline \multirow[t]{2}{*}{ IL-8 } & Yes & 16 & $15.44(10.25-56.46)$ & 0.449 & 8 & $15.44(10.95-51.21)$ & 0.764 \\
\hline & No & 6 & $12.79(8.72-21.6)$ & & 14 & $13.7(9.01-29.86)$ & \\
\hline \multirow[t]{2}{*}{$\mathrm{IL}-12$} & Yes & 21 & $4.01(2.81-5.82)$ & 0.989 & 13 & $4.47(3.35-5.94)$ & 0.519 \\
\hline & No & 8 & $4.54(2.95-5.60)$ & & 16 & $3.99(2.72-5.35)$ & \\
\hline \multirow[t]{2}{*}{ IL-10 } & Yes & 20 & $24.62(16.57-47.64)$ & 0.220 & 12 & $20.55(5.21-40.69)$ & 0.922 \\
\hline & No & 9 & $14.39(10.33-61.24)$ & & 17 & $24.37(13.52-51.11)$ & \\
\hline \multirow[t]{2}{*}{ IL-7 } & Yes & 22 & 18.16 (11.7-22.06) & 0.582 & 13 & $19.24(17.11-23.66)$ & 0.082 \\
\hline & No & 12 & $14.41(11.45-23.63)$ & & 21 & $13.99(10.38-21.45)$ & \\
\hline \multirow[t]{2}{*}{ IL-13 } & Yes & 19 & $27.47(20.06-37.07)$ & 0.159 & 13 & $29.9(21.79-38.55)$ & 0.205 \\
\hline & No & 9 & $17.68(13.24-34.71)$ & & 15 & $25.36(14.89-34.08)$ & \\
\hline
\end{tabular}

\#; analyzed by Mann-Whitney $U$ test at $<0.05$ level of statistical significance $p$ significant at $<0.05$ (2-tailed)

$I Q R$ interquartile range

$n$ number of samples

associated with increased pro-inflammatory cytokine responses including, TNF- $\alpha$, IL- 6 and IL- $8[5,8,10]$, and these cytokines are observed to play key roles in the pathogenesis of some CVDs [11]. Also, prolonged sedentary time could be associated with reduced anti- inflammatory cytokine responses including IL-10 [9]. This assertion is buttressed by the association of sedentary lifestyle with reduced peripheral circulation of a key anti-inflammatory molecule, adiponectin [5], and particularly, adiponectin induces the release of IL-10 by

Table 5 Comparison of serum cytokines levels across the different infection groups

\begin{tabular}{|c|c|c|c|c|c|}
\hline \multirow{2}{*}{$\begin{array}{l}\text { Parameters } \\
\text { (pg/mL) }\end{array}$} & \multicolumn{4}{|l|}{ Infection status } & \multirow[t]{2}{*}{$* P$} \\
\hline & Un-infected & P. falciparum mono-infection & HBV mono-infection & P. falciparum/HBV co-infection & \\
\hline \multicolumn{3}{|c|}{ Pro-inflammatory cytokines } & \multicolumn{2}{|l|}{ Median (IQR), $\mathrm{n}$} & \\
\hline TNF-a & $11.52(8.55-13.85) 11$ & $8.26(4.49-26.63) 4$ & $11.44(6.93-12.56) 9$ & $12.95(10.97-15.59) 9$ & 0.290 \\
\hline $\mathbb{I L}-1 \beta$ & $4.56(2.67-6.34) 9$ & $2.94(1.97-4.14) 3$ & $3.07(2.65-4.09) 6$ & $3.55(3.12-4.82) 7$ & 0.442 \\
\hline IL-6 & 37.09 (24.44-48.26) 11 & $32.13(19.09-165.8) 3$ & $28.70(22.68-39.35) 9$ & $34.52(37.59-55.42) 10$ & 0.686 \\
\hline IFN- $\gamma$ & $6.00(3.18-8.30) 10$ & 7.79 (3.22-16.00) 3 & $6.99(5.70-9.38) 8$ & $6.10(3.74-9.85) 9$ & 0.801 \\
\hline IL-8 & $47.38(7.71-395.00) 9$ & $10.17(5.51-20.22) 3$ & $12.99(10.54-19.48) 5$ & $15.93(9.15-23.60) 5$ & 0.546 \\
\hline $\mathbb{I L}-12$ & $2.97(1.82-5.02) 10$ & $3.87(2.87-4.00) 3$ & $4.54(3.75-5.12) 7$ & $5.68(3.93-6.32) 9$ & 0.154 \\
\hline \multicolumn{3}{|c|}{ Anti-inflammatory cytokines } & \multicolumn{2}{|l|}{ Median (IQR), n } & \\
\hline $\mathbb{I L}-10$ & $17.18(11.82-25.67) 9$ & $24.86(15.06-49.26) 3$ & $23.20(11.86-47.39) 8$ & $27.41(17-99.52) 9$ & 0.201 \\
\hline IL-7 & $16.67(11.33-21.49) 11$ & $10.40(6.70-18.81) 4$ & $17.54(11.85-23.66) 9$ & $19.50(16.79-25.00) 10$ & 0.190 \\
\hline $\mathbb{L L}-13$ & 19.65 (15.86-35.49) 9 & $27.47(25.36-41.54) 3$ & $25.55(13.11-38.54) 8$ & $31.50(21.60-35.35) 8$ & 0.763 \\
\hline
\end{tabular}

*; analyzed by Krulskal-Wallis test at $<0.05$ level of statistical significance $p$ significant at $<0.05$ (2-tailed)

$I Q R$ interquartile range

$n$ number of samples 
Table 6 Comparison of serum cytokines levels across sedentary and non-sedentary groups with different infections

\begin{tabular}{|c|c|c|c|c|c|}
\hline \multirow{2}{*}{$\begin{array}{l}\text { Parameters } \\
(\mathrm{pg} / \mathrm{mL})\end{array}$} & \multicolumn{4}{|c|}{ Sedentary groups with different infection status } & \multirow[t]{2}{*}{$* p$} \\
\hline & $\begin{array}{l}\text { Sedentary and Infected } \\
\text { group }\end{array}$ & $\begin{array}{l}\text { Sedentary and Un- } \\
\text { infected }\end{array}$ & $\begin{array}{l}\text { Non-sedentary and Infected } \\
\text { group }\end{array}$ & $\begin{array}{l}\text { Non-sedentary and Un-infected } \\
\text { group }\end{array}$ & \\
\hline \multicolumn{3}{|c|}{ Pro-inflammatory cytokines } & Median (IQR), n & & \\
\hline TNF-a & $12.95(10.03-15.59) 13$ & $10.72(8.55-12.89) 7$ & $9.84(6.45-11.92) 9$ & $12.69(7.93-14.51) 4$ & 0.349 \\
\hline $\mathrm{IL}-1 \beta$ & $3.35(2.91-4.67) 10$ & $3.91(1.98-9.58) 5$ & $3.14(2.36-4.59) 6$ & $4.56(3.62-5.44) 4$ & 0.615 \\
\hline IL-6 & $37.63(26.48-50.68) 13$ & $33.21(25.49-47.9) 8$ & $27.78(20.98-36.3) 9$ & $42.01(21.21-56.59) 3$ & 0.534 \\
\hline $\mathrm{IFN}-\gamma$ & $6.89(3.83-10.18) 17$ & $6.00(3.86-7.47) 8$ & $6.25(3.87-8.81) 12$ & $5.52(2.16-9.92) 4$ & 0.867 \\
\hline IL-8 & $13.6(10.25-19.34) 8$ & $47.38(7.60-395.00) 5$ & $15.93(7.36-22.12) 5$ & $35.95(5.36-404.4) 4$ & 0.685 \\
\hline $\mathrm{IL}-12$ & $4.68(3.78-5.83) 12$ & $4.12(2.75-5.86) 7$ & $4.27(3.87-5.96) 7$ & $1.99(1.80-3.54) 4$ & 0.119 \\
\hline \multicolumn{3}{|c|}{ Anti-inflammatory cytokines } & Median (IQR), n & & \\
\hline IL-10 & $34.4(23.94-69.84) 13$ & $12.66(11.82-35.28) 5$ & $16.49(14.39-27.41) 7$ & $17.37(11.53-29.71) 4$ & 0.098 \\
\hline IL-7 & $20.22(17.72-26.28) 7$ & $17.04(11.92-20.51) 8$ & $19.24(10.46-19.76) 3$ & $17.81(10.53-34.92) 4$ & 0.461 \\
\hline IL-13 & $30.14(25.00-36.64) 10$ & 19.65 (15.86-48.81) 5 & $27.47(14.63-38.20) 9$ & $23.26(15.70-34.90) 4$ & 0.852 \\
\hline
\end{tabular}

*; analyzed by Krulskal-Wallis test at $<0.05$ level of statistical significance

$p$ significant at $<0.05$ (2-tailed)

$I Q R$ interquartile range

$n$ number of samples

human leukocytes [25]. However, evidence shows that disruption of prolonged sitting with short bouts of physical activity has beneficial effects including reducing inflammatory mediators in individuals $[8,26]$. The participants in this study, though are a predominantly sedentary group, their sitting time may be intermittently interrupted as they get up to sell items and perform some mobile trading activities. Therefore, it is possible that such mobility may account for the similarity in cytokine responses among the traders categorized into the different sedentary lifestyle.

The study reports a $53.8 \%$ prevalence of hypertension among the traders, which is higher than the range of 19.0 to $48.0 \%$ reported nationally [27]. The evidence of hypertension among the traders corroborates the finding of a previous study conducted among market men and women in the Tamale metropolis of Ghana [3]. What is worrying from this study is the evidence that majority of the participants' resort to self-medication when they felt bodily and chest pains; which are known symptoms of hypertension. There is therefore the need for concerted efforts to raise knowledge and awareness of hypertension among predominantly sedentary traders in the Tamale Metropolis of Ghana. Though hypertension was common among the participants, it was independent of $P$. falciparum and/or HBV infections, which may lend support to the evidence that have shown no association between P. falciparum or HBV with CVDs [14, 15]. Unlike the study that showed an imbalance in serum pro- and anti-inflammatory cytokine responses in individuals with hypertension [28], this current study observed a contrary finding. We speculate that differences in the etiology of hypertension and population-specific factors may contribute to our current finding.

The prevalence of anemia (44.5\%) reported among the traders is slightly higher than the national rate of $42.0 \%$ previously reported for non-pregnant/non-breastfeeding individuals [29]. Evidence shows that poorer or lack of exercise is a risk factor for anemia [30], which may explain our finding of a substantial number of the traders with anemia, since most of the traders spent longer hours sitting. This anemia may lead to prolonged sedentary time among the traders as they are likely to feel lethargic upon exertion. It has also been reported that anemia is a marker of an increased risk for hypertension and CVD-related deaths [31, 32]. Much as this call for caution among sedentary traders, there is the need for routine diagnosis of anemia among risks groups including traders, so as to avert the possible risks of developing CVDs and subsequent death.

Though $P$. falciparum and HBV majorly trigger the release of the pro-inflammatory cytokines [16], the infections appeared not to markedly influence the inflammatory responses in the traders. It has been suggested that adults with $P$. falciparum infection show controlled inflammatory responses due to increased history of exposure, and that higher parasite loads are required to elicit the inflammatory responses and cause disease symptoms [33]. HBV infection on the other hand has different phases, and during the immune tolerance phase, the host elicits no vigorous inflammatory response to the infection and so no disease symptoms are observed [13]. It is very likely that the traders who were domiciled in an endemic region for both conditions were 
historically increasingly exposed and were also more likely to be in the immune tolerance phase for the two conditions respectively, and this could account for the independence of infections on the cytokine responses.

\section{Limitation}

A smaller sample size was used and our observation should be replicated in a substantially larger population. The measurement of sedentary behaviour was based on self-report, per the questionnaire administered. Consequently, the use of accelerometers could have offered an increased accuracy in the measurement of sedentary time. Nonetheless, time spent in self-reported sedentary behaviour has been recognized as a unique risk factor for several cardio-metabolic diseases [10, 34]. Among the study participants, the stage of HBV or P. falciparum infection was not known. The study described the infections as asymptomatic based on the results of the RDTs and PCRs, and coupled with the observations that the study participants went about their routine activities with no experience of disease symptoms.

\section{Conclusion}

Our data suggest that asymptomatic infections of $P$. falciparum and HBV as well as a high prevalence of hypertension and anemia had no significant impact on cytokine response profiles among predominantly sedentary traders in Tamale metropolis of Ghana. In addition, the infections did not influence the occurrence of hypertension.

\section{Supplementary Information}

The online version contains supplementary material available at https://doi. org/10.1186/s12879-020-05692-2.

Additional file 1. Socio-demographic characteristics of the study participants $(n=400)$.

Additional file 2. Behavioral/life style determinants in study participants $(n=400)$.

\section{Abbreviations}

BP: Blood pressure; CVDs: Cardiovascular diseases; Hb: Hemoglobin; HbsAg: Hepatitis B surface antigen; HBV: Hepatitis B virus; IFN- $\gamma$ : Interferon gamma; IL: Interleukin; IQR: Interquartile range; PCR: Polymerase chain reaction; RDTs: Rapid diagnostic tests; TNF-a: Tumor necrosis factor alpha

\section{Acknowledgments}

We are most grateful to Mr. Victor Sadongo, Mr. Misbao Muntari, Ms. Yvette Duobu and Ms. Zaheeda Millie of the Department of Biochemistry and Applied Chemistry, University for Development Studies, for assisting in screening of participants, and acquisition and entry of the data. We also extend our gratitude to Professor Gordon Awandare, Director of WACCBIP, University of Ghana, for providing resources for the PCRs and cytokine assays.

\section{Authors' contributions}

NGA contributed in: conception, study design, laboratory investigation, data acquisition, data analysis and interpretation, and drafting the article. PAA contributed in: study design, curation of data and analysis, and critically revising the draft manuscript. ZZ contributed in: data acquisition and curation, and screening of participants. JS contributed in: curation of data, and Laboratory investigation. FA contributed in: curation of data, and Laboratory investigation. GKH contributed in: conception, study design, curation of data and analysis, critically revising the draft manuscript. All authors read and approved the final article.

Funding

Not applicable.

Availability of data and materials

The datasets supporting the findings of this article are available in this manuscript.

Ethics approval and consent to participate

The study was approved by the joint ethical review board (ERB) of the School of Medicine and Health Sciences/School of Allied Health Sciences (SMHS/SAHS) of the University for Development Studies, Ghana. Permission was given by the leadership of the traders' union, Tamale-Ghana, before the data were collected. Written informed consent was obtained from the study subjects prior to data collection. Prior to obtaining consent, the participants were provided with information about the purpose, benefits and associated risk of the study. The participants were also informed that their participation was voluntary and that they could withdraw from the study at any time without consequences. In addition, they were assured that all responses received would be kept confidential. They were then asked to sign or thumbprint a written consent form before participating in the study.

\section{Consent for publication}

Not applicable.

\section{Competing interests}

The authors declare that there are no conflicts of interest.

\section{Author details}

${ }^{1}$ West African Centre for Cell Biology of Infectious Pathogens (WACCBIP), University of Ghana, P. O. Box LG 54, Legon, Accra, Ghana. ${ }^{2}$ Department of Biochemistry \& Molecular Medicine, School of Medicine, University for Development Studies, P. O. Box TL, 1883 Tamale, Ghana. ${ }^{3}$ Department of Nutritional Sciences, School of Allied Health Sciences, University for Development Studies, P. O. Box TL, 1883 Tamale, Ghana.

Received: 7 August 2020 Accepted: 8 December 2020 Published online: 14 December 2020

\section{References}

1. Tarp J, Brønd JC, Andersen LB, Møller NC, Froberg K, Grøntved A. Physical activity, sedentary behavior, and long-term cardiovascular risk in young people: a review and discussion of methodology in prospective studies. J Sport Health Sci. 2016;5(2):145-50.

2. Guedes DP, Guedes JERP, Barbosa DS, Oliveira JAd, Stanganelli LCR: Cardiovascular risk factors in adolescents: biological and behavioral indicators. Arq Bras Cardiol 2006, 86(6):439-450.

3. Yakong V, Dapare P, Boateng B, Shittu S, Ziba F, Sakyi-Djan I, Tiah H, Baidoo F. Obesity and hypertension among market men and women in the tamale metropolis. J Med Biomed Sci. 2015;4(3):9-17.

4. Thorp AA, Owen N, Neuhaus M, Dunstan DW. Sedentary behaviors and subsequent health outcomes in adults: a systematic review of longitudinal studies, 1996-2011. Am J Prev Med. 2011;41(2):207-15.

5. Allison MA, Jensky NE, Marshall SJ, Bertoni AG, Cushman M. Sedentary behavior and adiposity-associated inflammation: the multi-ethnic study of atherosclerosis. Am J Prev Med. 2012;42(1):8-13.

6. Falconer C, Cooper A, Walhin J, Thompson D, Page A, Peters T, Montgomery A, Sharp D, Dayan C, Andrews R. Sedentary time and markers of inflammation in people with newly diagnosed type 2 diabetes. Nutrition Metabolism Cardiovasc Dis. 2014;24(9):956-62.

7. Healy GN, Matthews CE, Dunstan DW, Winkler EA, Owen N. Sedentary time and cardio-metabolic biomarkers in US adults: NHANES 2003-06. Eur Heart J. 2011;32(5):590-7. 
8. Dogra S, Wolf M, Jeffrey MP, Foley RC, Logan-Sprenger $H$, Jones-Taggart $H$, Green-Johnson JM. Disrupting prolonged sitting reduces IL-8 and lower leg swell in active young adults. BMC Sports Sci Med Rehabil. 2019;11(1):23.

9. Nishida M, Moriyama T, Sugita Y, Yamauchi-Takihara K. Interleukin-10 associates with adiponectin predominantly in subjects with metabolic syndrome. Circ J. 2007;71(8):1234-8.

10. Yates T, Khunti K, Wilmot EG, Brady E, Webb D, Srinivasan B, Henson J, Talbot D, Davies MJ. Self-reported sitting time and markers of inflammation, insulin resistance, and adiposity. Am J Prev Med. 2012;42(1):1-7.

11. Yudkin JS, Kumari M, Humphries SE, Mohamed-Ali V. Inflammation, obesity, stress and coronary heart disease: is interleukin- 6 the link? Atherosclerosis. 2000;148(2):209-14.

12. Heinemann M, Phillips RO, Vinnemeier CD, Rolling C, Tannich E, Rolling T. High prevalence of asymptomatic malaria infections in adults, Ashanti Region, Ghana, 2018. Malar J. 2020;19:366. https://doi.org/10.1186/s12936020-03441-z.

13. Tran $\Pi$. Immune tolerant hepatitis B: a clinical dilemma. Gastroenterol Hepatol. 2011;7(8):511.

14. Kuo C-S, Chen Y-T, Hsu C-Y, Chang C-C, Chou R-H, Li S-Y, Kuo S-C, Huang P$\mathrm{H}$, Chen J-W, Lin S-J. The impact of chronic hepatitis B infection on major adverse cardiovascular events and all-cause mortality in patients with diabetes: a nationwide population-based study from Taiwan. BMJ Open. 2017;7(8):e016179.

15. Wijarnpreecha $K$, Thongprayoon $C$, Panjawatanan $P$, Ungprasert $P$. Hepatitis $B$ virus infection and risk of coronary artery disease: a meta-analysis. Ann Translational Medicine. 2016;4(21). https://doi.org/10.21037/atm.2016.11.12.

16. Anabire NG, Aryee PA, Abdul-Karim A, Quaye O, Awandare GA, Helegbe GK: Impact of malaria and hepatitis B co-infection on clinical and cytokine profiles among pregnant women. PLoS One 2019, 14(4).

17. Virtanen M, Heikkilä K, Jokela M, Ferrie JE, Batty GD, Vahtera J, Kivimäki M. Long working hours and coronary heart disease: a systematic review and meta-analysis. Am J Epidemiol. 2012;176(7):586-96.

18. Petry NM. A comparison of young, middle-aged, and older adult treatmentseeking pathological gamblers. Gerontologist. 2002:42(1):92-9.

19. The Seventh Report of the Joint National Committee on Prevention, Detection, Evaluation, and Treatment of High Blood Pressure. Bethesda (MD): National Heart, Lung, and Blood Institute (US); 2004 Aug. Table 3, Classification of blood pressure for adults. [https://www.ncbi.nlm.nih.gov/ books/NBK9633/table/A32/].

20. World Health Organization: Haemoglobin concentrations for the diagnosis of anaemia and assessment of severity. 2011.

21. Snounou G, Viriyakosol S, Jarra W, Thaithong S, Brown KN. Identification of the four human malaria parasite species in field samples by the polymerase chain reaction and detection of a high prevalence of mixed infections. Mol Biochem Parasitol. 1993;58(2):283-92.

22. Nsobya SL, Parikh S, Kironde F, Lubega G, Kamya MR, Rosenthal PJ, Dorsey G. Molecular evaluation of the natural history of asymptomatic parasitemia in Ugandan children. J Infect Dis. 2004;189(12):2220-6.

23. Garson J, Grant P, Ayliffe U, Ferns R, Tedder R. Real-time PCR quantitation of hepatitis $B$ virus DNA using automated sample preparation and murine cytomegalovirus internal control. J Virol Methods. 2005;126(1):207-13.

24. Anabire NG, Aryee PA, Abdul-Karim A, Abdulai IB, Quaye O, Awandare GA, et al. Prevalence of malaria and hepatitis $B$ among pregnant women in northern Ghana: comparing RDTs with PCR. PLoS One. 2019;14(2):e0210365. https://doi.org/10.1371/journal.pone.0210365.

25. Wolf AM, Wolf $D$, Rumpold $H$, Enrich B, Tilg H. Adiponectin induces the antiinflammatory cytokines IL-10 and IL-1RA in human leukocytes. Biochem Biophysical Res Communications. 2004;323(2):630-5.

26. Grace MS, Formosa MF, Bozaoglu K, Bergouignan A, Brozynska M, Carey AL, Veiga CB, Sethi P, Dillon F, Bertovic DA. Acute effects of active breaks during prolonged sitting on subcutaneous adipose tissue gene expression: an ancillary analysis of a randomised controlled trial. Sci Rep. 2019;9(1):1-11.

27. Bosu WK. Epidemic of hypertension in Ghana: a systematic review. BMC Public Health. 2010;10(1):418.

28. Mirhafez SR, Mohebati M, Disfani MF, Karimian MS, Ebrahimi M, Avan A, Eslami S, Pasdar A, Rooki H, Esmaeili H. An imbalance in serum concentrations of inflammatory and anti-inflammatory cytokines in hypertension. J Am Soc Hypertension. 2014;8(9):614-23.

29. MacDonald C, Namarika R, Yiannakis M: Anemia_can its widespread prevalence among women in developing countries be impacted. Table 4 :
Anemia Prevalence Rates in Women, Selected Countries (by WHO region) 2002:5.

30. Ekegren CL, Beck B, Climie RE, Owen N, Dunstan DW, Gabbe BJ. Physical activity and sedentary behavior subsequent to serious orthopedic injury: A systematic review. Archives Physical Med Rehabil. 2018;99(1):164-77 e166.

31. Lee W-C, Fang H-Y, Chen H-C, Chen C-J, Yang C-H, Hang C-L, et al. Anemia: A significant cardiovascular mortality risk after ST-segment elevation myocardial infarction complicated by the comorbidities of hypertension and kidney disease. PLoS One. 2017;12(7):e0180165. https://doi.org/10.1371/ journal.pone.0180165.

32. Paul B, Wilfred NC, Woodman R, DePasquale C. Prevalence and correlates of anaemia in essential hypertension. Clin Exp Pharmacol Physiol. 2008;35(12): 1461-4.

33. Ademolue TW, Awandare GA. Evaluating antidisease immunity to malaria and implications for vaccine design. Immunology. 2018;153(4):423-34

34. Owen N, Healy GN, Matthews CE, Dunstan DW. Too much sitting: the population-health science of sedentary behavior. Exerc Sport Sci Rev. 2010; 38(3):105.

\section{Publisher's Note}

Springer Nature remains neutral with regard to jurisdictional claims in published maps and institutional affiliations.
Ready to submit your research? Choose BMC and benefit from:

- fast, convenient online submission

- thorough peer review by experienced researchers in your field

- rapid publication on acceptance

- support for research data, including large and complex data types

- gold Open Access which fosters wider collaboration and increased citations

- maximum visibility for your research: over $100 \mathrm{M}$ website views per year

At $\mathrm{BMC}$, research is always in progress.

Learn more biomedcentral.com/submissions 